than oesophageal cancer $(0.735$ vs. $0.900, \mathrm{p}<0.001)$. Inflammatory scores in GOJ cancers were lower than in gastric cancer and higher than in oesophageal cancer.

Conclusion This study provides direct evidence for marked differences in the gastric mucosal phenotype in the patients with oesophageal versus gastric non-cardia cancer, with the former being healthy and uninflamed, but the latter atrophic and inflamed. The background gastric mucosa of GOJ cancer supported them being two distinct aetiologies, one group resembling oesophageal adenocarcinoma and other gastric non-cardia cancer.

Disclosure of Interest None Declared.

\section{PTU-165 WORLDWIDE EPIDEMIOLOGICAL EVIDENCE SUPPORTS A COMMON FACTOR PREDISPOSING TO NON-CARDIA GASTRIC CANCER AND PROTECTING FROM OESOPHAGEAL ADENOCARCINOMA}

${ }^{1} \mathrm{MH}$ Derakhshan* ${ }^{2} \mathrm{DH}$ Brewster, ${ }^{3} \mathrm{Il}$ Going, ${ }^{1} \mathrm{EV}$ Robertson, ${ }^{4} \mathrm{M}$ Arnold ${ }^{4} \mathrm{D}$ Forman, ${ }^{1} \mathrm{KE}$ McColl. IInstitute of Cardiovascular and Medical Sciences, University of Glasgow, Glasgow, UK; ${ }^{2}$ Scottish Cancer Registry, NHS UK, Edinburgh, UK; ${ }^{3}$ Institute of Cancer Sciences, University of Glasgow, Glasgow, UK; ${ }^{4}$ Division of Cancer Information, International Agency for Research on Cancer, Lyon, France

\subsection{6/gutjnl-2014-307263.239}

Introduction During last three decades, global incidence of oesophageal adenocarcinoma has increased more rapidly than any other cancer. A concurrent reduction in the incidence of gastric cancer has been reported from some populations. We aimed to examine the geographical pattern of oesophageal adenocarcinoma versus gastric non-cardia cancer across the world where reliable cancer registry data were available.

Methods Data were abstracted from "Cancer Incidence in Five Continents" Volume 10. Oesophageal and gastric cancers were selected based on ICD-10 codes C15 and C16, respectively. Oesophageal adenocarcinomas were identified by ICD-O morphology codes. Datasets reporting $>500$ cases for total gastric cancer and $>100$ for total oesophageal cancer were selected. We examined correlation between age-standardised Incidence rates (ASR) of oesophageal adenocarcinoma and non-cardia gastric cancer using Spearman's non-parametric correlation coefficient (CC). We also allocated cardia cancers into oesophageal and non-cardia gastric adenocarcinoma categories based on gender ratio for oesophageal adenocarcinoma and non-cardia gastric cancer in each dataset.

Results Out of 424 datasets from 290 cancer registries, 206 datasets covering 40 countries met the selection criteria. There was a strong inverse correlation between oesophageal adenocarcinoma and gastric non-cardia cancer in males $(\mathrm{CC}=-0.768, \mathrm{p}<0.001)$ and females $(\mathrm{CC}=-0.705, \mathrm{p}<0.001)$. After dividing cardia cancer into two subtypes with potentially oesophageal or gastric origin and adding them to original oesophageal adenocarcinoma or gastric non-cardia groups, the inverse correlation remained strong in males $(\mathrm{CC}=-0.660, \mathrm{p}<0.001)$ and females $(\mathrm{CC}=-0.536, \mathrm{p}$ $<0.001)$. Oesophageal adenocarcinoma only showed a rise when incidence of non-cardia gastric cancer fell below 9/100,000 person-years for males and 4.5/100,000 person-years for females. Conclusion This cross-sectional study is consistent with a common underlying factor predisposing to non-cardia gastric cancer and protecting from oesophageal adenocarcinoma, such as $\mathrm{H}$. pylori atrophic gastritis. If this is the case, then the incidence of non-cardia gastric cancer would need to fall to substantially lower levels than currently seen in Far Eastern populations before any rise in oesophageal adenocarcinoma would be apparent.

Disclosure of Interest None Declared.
PTU-166 DETECTION RATES OF GASTRIC CANCER AT THE QUEEN ELIZABETH HOSPITAL BIRMINGHAM 2009-2013

N Sagar*, N Bhala. Gastroenterology, Queen Elizabeth Hospital Birmingham, Birmingham, UK

\subsection{6/gutjnl-2014-307263.240}

Introduction Despite open-access endoscopy, previous series have suggested that between $8-20 \%$ of early gastric cancers (GC) are potentially missed at prior endoscopy. ${ }^{1,2}$ Although upper gastrointestinal alarm symptoms are more frequently associated with malignancy, this may represent advanced cancer with poorer survival rates, as patients with early GCs may be asymptomatic. The false-negative rate for the diagnosis of GC may also be a measure of quality for endoscopy services. This is based on a reported median duration of 37 months between endoscopic diagnosis of early GC and progression to advanced GC, ${ }^{2,3}$ so we assessed all oesophagogastroduodenoscopy (OGD) findings to assess detection of GC in a large tertiary hospital in the West Midlands.

Methods Patients with histologically confirmed GC were identified from histopathology and endoscopy records. Patients who had undergone at least one OGD before the diagnosis were studied. Detection of GC within 3 years of a negative OGD was interpreted as a false negative.

Results Between September 2009 and September 2013, 16823 OGDs were performed. GC was diagnosed in 75 (0.45\%) patients (male/female ratio 1.78; median age 74; $85 \%$ Caucasian). Sixty-seven (89\%) of the 75 patients with GC presented with alarm symptoms. 33\% (25) were done as inpatients, with $43 \%$ (at least 32 of 50 outpatients) being referred as urgent outpatients. Five of the $75(7 \%)$ patients had previous OGDs within three years preceding diagnosis. Only one of these was planned because of a suspicious gastric ulcerative lesion at the same site, with other causes being gastric polyps (2); normal (1) and gastritis (1). There were $53(71 \%)$ deaths in total, $47(89 \%)$ of these patients had alarm symptoms at diagnosis of GC.

Conclusion The absolute rates of GC are low $(0.1 \% / \mathrm{OGD} /$ year) and false-negative rates of 5\% (within 3 years) for diagnosis of GC are reassuring with only a minority of preceding OGDs in this series demonstrating suspicious lesions. Whilst GC presents with alarm symptoms in the vast majority, the prognosis remains very poor, so continued quality measures in endoscopy will be required to ensure that early gastric cancers are not missed.

\section{REFERENCES}

1 Vradelis S, Maynard N, Warren BF et al. Quality control in upper gastrointestinal endoscopy: detection rates of gastric cancer in Oxford 2005-2008. Postgrad Med J. 2011 May:87(1027):335-9

2 Hosokawa O, Tsuda S, Kidani E, et al. Diagnosis of gastric cancer up to three years after negative upper gastrointestinal endoscopy. Endoscopy 1998:30:66974

3 Tsukuma H, Mishima T, Oshima A. Prospective study of "early" gastric cancer. Int J Cancer 1983:31:421-6

Disclosure of Interest None Declared.

\section{PTU-167 BARRETT'S OESOPHAGUS SURVEILLANCE STUDY (BOSS) UPDATE: SUCCESSFUL RECRUITMENT TO A LONG FOLLOW-UP RCT}

OJ Old*, C Stokes, S Woods, C Foy, J Hapeshi, H Barr on behalf of on behalf of the BOSS team. Gloucestershire Hospitals NHS Trust, Gloucester, UK

10.1136/gutjnl-2014-307263.241 
Introduction Current BSG guidelines advise routine endoscopic surveillance for patients with Barrett's oesophagus to enable early detection of oesophageal adenocarcinoma. However, evidence for improved outcomes from surveillance is weak and remains the subject of debate. To address these concerns, BOSS aims to compare the benefits of 2-yearly surveillance endoscopy against endoscopy on an 'at need' basis only. As a large, multicentre trial with 10 year follow-up, this ambitious project faced a number of challenges to recruitment, particularly the acceptability of randomising to a control arm with no routine endoscopy, especially in sites where surveillance had been established.

Methods Multi-centre randomised controlled trial (ISRCTN54190466). Inclusion criteria: patients over 18 with endoscopic and histologically proven Barrett's oesophagus $>1$ $\mathrm{cm}$. Exclusion criteria: patients unable to consent, unfit for endoscopy, high-grade dysplasia or cancer, or participation in AspECT trial. Target sample size: 3400. Recruitment: patients identified at local centres with new diagnosis, or existing diagnosis of Barrett's and endoscopy within last 2 years. Follow-up will be for 10 years. Intervention arm will receive 2-yearly surveillance endoscopy, control arm will receive endoscopy on an 'at need' basis if symptomatic. Primary outcome: all cause mortality. Results Recruitment began March 2009, target of 3400 was reached ahead of schedule in October 2011. 3469 consented to be randomised (58.4\% of eligible screened patients): 1739 in 2yearly surveillance, 1730 in 'at need' arm. 127 hospitals were open to recruitment, though 3 withdrew from the study, recruitment was closed in one centre by the trial team, and 11 did not recruit any patients. 3780 screened patients did not enter: 1309 ineligible, 2471 declined. Most common reasons for patients to decline were: preference for surveillance, preference not to have endoscopies and a family history of cancer. As of January 2014, 111 patients were no longer in their originally randomised treatment groups: 55 due to patient/clinician decision, 56 for other reasons. For 107 of those 111 patients, data continues to be collected.

Conclusion The successful recruitment to BOSS strongly supports the acceptability of randomisation to the control arm, and patients' willingness to participate in a long follow-up RCT. The large number of centres and enthusiastic principal investigators have shown continued success, and great promise for BOSS to answer key questions on Barrett's surveillance.

\section{REFERENCE}

Fitzgerald RC, et al. Gut 2014:63:7-42

Disclosure of Interest None Declared.

\section{PTU-168 BARRETT'S OESOPHAGUS SCREENING: INFRARED SPECTROSCOPY FOR CYTOLOGICAL ASSESSMENT}

${ }^{1}$ OJ Old*, ${ }^{1} \mathrm{M}$ Almond, ${ }^{1} \mathrm{G}$ Lloyd, ${ }^{2} \mathrm{D}$ Townsend, ${ }^{2} \mathrm{~K}$ Lenau, ${ }^{2} \mathrm{M}$ Diem, ${ }^{1} \mathrm{H}$ Barr. ${ }^{1}$ Gloucestershire Hospitals NHS Trust, Gloucester, UK; ${ }^{2}$ Northeastern University, Boston, USA

\subsection{6/gutjnl-2014-307263.242}

Introduction Screening for Barrett's oesophagus could allow early detection, enabling timely diagnosis and intervention for oesophageal adenocarcinoma. Recent studies have shown the acceptability of a swallowed cytology brush ('Cytosponge') for cell collection. If introduced, cytological assessment would pose several challenges. Firstly, oesophageal cytology is performed infrequently, and expertise in this field is correspondingly
Abstract PTU-168 Table 1

\begin{tabular}{|c|c|c|c|}
\hline & Normal squamous & Barrett's oesoph. & Dysplasia \\
\hline Sensitivity & $100 \% \quad 1 \%$ & $96 \% \quad 2 \%$ & $94 \% 3 \%$ \\
\hline Specificity & $99 \% \quad 1 \%$ & $99 \% \quad 1 \%$ & $98 \% 1 \%$ \\
\hline
\end{tabular}

limited. Secondly, assessment of individual cells is challenging even for experienced cytopathologists, with a degree of interobserver variability. Thirdly, screening would require a great deal of cytopathology resources. Infrared spectroscopy (IR) gives reproducible spectra based on cell biochemistry; applying multivariate statistical analysis and computer modelling can provide robust and rapid discrimination between pathological cell subtypes. We aimed to demonstrate the potential application of IR in analysis of oesophageal cytology.

Methods Endoscopic cytology brushes were used to collect oesophageal cells from patients undergoing endoscopy for Barrett's oesophagus. Cells were fixed in formalin, centrifuged and slides prepared. IR spectra were measured across the entire sample area. Pre-processing steps allowed spectra from individual cells to be reconstituted. Further pre-processing removed confounding effects and enhanced signal-to-noise ratios. Conventional cytology analysis was undertaken to provide a reference for developing a predictive model using IR data. Chemometric analysis was then undertaken using Partial Least Squares Discriminant Analysis (PLS1DA) and cross-validation performed.

Results 23 cytology brush samples were collected from 11 patients. 4 samples contained low cell counts and were excluded from analysis. 5536 cells (2339 normal squamous, 2511 Barrett's oesophagus and 686 dysplastic) were used to create and validate a predictive model. The predictive capability of the model is shown in the table below:

Conclusion The high accuracy demonstrated by our predictive model suggests IR is a promising candidate for cytological analysis of oesophageal cells. As an objective, automated system, this technique could prove invaluable for Barrett's screening in future.

\section{REFERENCE}

Schubert JM et al. Lab Invest. 2010;90(7):1068-1077

Disclosure of Interest None Declared.

\section{PTU-169 LONG-LASTING OESOPHAGEAL MUCOSAL PROTCTION WITH ALGINATES: A POTENTIAL FOR TOPICAL MUCOSAL THERAPY IN GASTRO-OESOPHAGEAL REFLUX}

${ }^{1} \mathrm{P}$ Woodland*, ${ }^{1} \mathrm{C}$ Lee, ${ }^{2} \mathrm{P}$ Dettmar, ${ }^{1} \mathrm{SL}$ Preston, ${ }^{1} \mathrm{D}$ Sifrim. ${ }^{1}$ Barts and the London School of Medicine and Dentistry, Queen Mary University of London, London; ${ }^{2}$ Technostics Limited, Hull, UK

\subsection{6/gutjpl-2014-307263.243}

Introduction Patients with non-erosive reflux disease (NERD) exhibit impaired oesophageal mucosal barrier integrity in the form of dilated intercellular spaces and low transepithelial electrical resistance (TER). Such refluxate-induced changes to the mucosal integrity may underlie increased sensitivity to perception of reflux events, even on PPI, and could potentially be modified by application of topical solutions.

Sodium alginate solutions are used in treatment of GORD, with proposed mechanisms of action including acid buffering, displacement of the gastric acid pocket, and reduction of reflux events. We have recently described that in vitro topical 